\title{
Máscara de espalhamento e precipitação para os canais microondas do satélite NOAA
}

\author{
João C. Carvalho' ${ }^{1}$, Nelson J. Ferreira ${ }^{1}$, Fernando M. Ramos ${ }^{1}$, Lydie Lavanant ${ }^{2}$ \\ 1 INPE. Av. Astronautas, 1758, Jardim da Granja, CEP 12227-010, São José dos Campos, SP. Fone: (12) 3945-6534. \\ E-mail: jcarlos@ltid.inpe.br (Foto), fernando@lac.inpe.br, nelson@ltid.inpe.br \\ 2 CMS/Meto-France, Avenue de Lorraine, 147, CEP 22302, Lannion, France. E-mail: Iydie.lavanant@meteo.fr
}

Protocolo 139 - 5/9/2003 - Aprovado em 5/5/2005

\begin{abstract}
Resumo: Propõe-se, com o presente trabalho, avaliar uma metodologia para identificação de pixels contaminados por precipitação e/ou espalhamento utilizando-se dados dos canais do Advanced Microwave Sensor Unit (AMSU). A aplicação de metodologias desse tipo é útil para a inferência de perfis verticais de temperatura e umidade no Brasil, em situações de céu coberto. A validação dos resultados foi feita com base em um estudo de caso, em que se aplicou uma análise subjetiva, tomando-se como modelo a comparação com imagens das bandas infravermelho, visível e microondas. Os resultados mostraram excelente concordância entre os topos de nuvens com temperaturas de brilho baixas, afetadas pelo efeito de espalhamento devido à presença de chuva e gelo, e as áreas identificadas pelo algoritmo como sendo contaminadas por este efeito. $O$ algoritmo conseguiu identificar adequadamente os locais sob influência de precipitação e/ou espalhamento.
\end{abstract}

Palavras-chave: sondagens remotas, nuvens, ATOVS

\section{Scattering and rainfall mask for microwave channels of NOAA satellite}

\begin{abstract}
This work presents a methodology to identify precipitation and/or scattering pixels in the Advanced Microwave Sensor Unit (AMSU) channels. This procedure is useful for applications in atmospheric temperature and moisture retrievals over Brazil under cloudy sky conditions. A subjective analysis based on a case study involving comparisons with infrared, visible and microwave images was applied for validation purpose. The results show an excellent relationship of cloud tops with low brightness temperature affected by scattering due to water drops and ice and the areas identified by the algorithm as being influenced by precipitation and/or scattering effect.
\end{abstract}

Key words: satellite soundings, clouds, ATOVS

\section{INTRODUÇÃO}

A partir de 1998, com o lançamento do satélite de órbita polar NOAA-15, tornou-se disponível uma nova geração de sensores para obtenção de perfis verticais de temperatura e umidade, o sistema Advanced TIROS Operational Vertical Sounder (ATOVS) (Goodrun et al., 2001), uma evolução do seu antecessor TOVS. São três os instrumentos que integram o sistema ATOVS: o High-Resolution Infrared Radiation Sounder (HIRS), composto de 20 canais espectrais na faixa espectral do infravermelho; o AMSU-A, com 15 canais microondas e o AMSU-B, composto de 5 canais também localizados na faixa espectral de microondas, e o Advanced Very High Resolution Radiometer (AVHRR/3), um imageador de alta resolução espacial $(1 \mathrm{~km})$ com 6 canais localizados nas faixas do visível e infravermelho.

A sondagem remota da atmosfera permite, a partir de um conjunto de radiâncias espectrais, determinar a estrutura vertical de temperatura, umidade e concentração de constituintes atmosféricos (Carvalho et al., 1999). Um dos grandes problemas relacionados a sondagens remotas da atmosfera é a obtenção de perfis verticais de temperatura e umidade sob a presença total ou parcial de nuvens, fato particularmente importante nas regiões tropicais, onde a nebulosidade é maior. Os novos instrumentos do sistema ATOVS têm grande potencial para melhorar a qualidade das sondagens nessas condições, devido ao aumento considerável de canais operando na faixa espectral das microondas (Smith, 1991). O impacto que a presença de 
nuvens e a precipitação causam na temperatura de brilho observada nestes canais, também é maior e, por conseqüência, modelos mais aprimorados de detecção de nuvens e precipitação devem ser utilizados.

$\mathrm{O}$ efeito do espalhamento nos canais AMSU causa diminuição da temperatura de brilho do pixel em relação àqueles não afetados, uma vez que as partículas de gelo espalham a radiação, diminuindo a quantidade que chega ao sensor. Por outro lado, chuva e água líquida nas nuvens agem como emissores quase não polarizados, de modo que sobre alvos frios e polarizados (oceanos, por exemplo) a chuva e água líquida nas nuvens tendem a despolarizar e aumentar a temperatura de brilho observada pelo satélite. Este efeito pode ser notado especialmente em baixas frequências $(23,8$ e 31,4 GHz) e seu impacto é limitado aos oceanos. Sobre o continente, onde a emissividade da superfície geralmente é alta, o impacto do sinal desses emissores atmosféricos normalmente é muito baixo (Labrot et al., 2003; English et al., 1999a). A aplicação das observações na faixa das microondas para o processo de inversão, complementa o uso dos canais infravermelhos, fornecendo informações, mesmo em situações com nebulosidade; e contudo, embora na faixa das microondas e dependendo do comprimento de onda, o fluxo de radiação pode interagir com nuvens e precipitação e, conseqüentemente, a aplicação de um controle de qualidade para observações microondas se faz tão necessário quanto aquele realizado para os radiômetros infravermelhos, porém, enquanto o controle de qualidade dos canais infravermelhos pode ser realizado apenas para distinguir entre pixel nublado, não nublado ou parcialmente nublado, para a radiometria microondas existe uma divisão mais sutil entre insignificantemente nublado e significantemente nublado, em que a definição de significância dependerá da freqüência do canal (English et al., 1999b). Isto significa que, ao contrário da radiação infravermelha, onde geralmente as nuvens podem ser consideradas totalmente opacas, para os canais microondas é a composição da nuvem que determina se um canal sofrerá ou não, algum tipo de interferência. Motivados por essa problemática, o presente trabalho propõe e avalia uma nova metodologia para identificação de pixels contaminados por precipitação e/ou espalhamento, utilizando-se dados dos canais AMSU. A aplicação de metodologias desse tipo pode contribuir significativamente para a inferência de perfis de temperatura e umidade no Brasil, em situações de céu coberto (Carvalho, 2002).

\section{MATERIAL E MÉTODOS}

Neste estudo foram usadas órbitas do satélite NOAA-15 transmitidas no formato HRPT pela estação de recepção do INPE, localizada em $22^{\circ} 7^{\prime}$ S e $45^{\circ} 0^{\prime} \mathrm{W}$, na cidade de Cachoeira Paulista, SP, Brasil. O NOAA-15 passa por um mesmo local duas vezes ao dia, ou seja, passagens matutinas (em torno de 10:30 UTC) e noturnas (em torno de 22:30 UTC). A metodologia aqui abordada se aplica também aos novos satélites da série NOAA.

O modelo ATOVS e AVHRR Processing Package (AAPP) foi usado para realizar a ingestão e o pré-processamento dos dados HRPT (Klaes, 1997; Klaes \& Scharaidt, 1999). Este procedimento resulta em dados calibrados de temperatura de brilho para todos canais ATOVS, localizado em coordenadas terrestres (latitude e longitude) e mapeados para uma grade de resolução comum. Salienta-se que foram realizadas todas as correções necessárias às aquisições HRPT NOAA-15, sobretudo aquelas referentes aos problemas do instrumento AMSU-B (Chalfant \& Reale, 1999).

Por estar apenas indiretamente associado à região contendo precipitação, o sinal proveniente de espalhamento pode ser observado tanto sobre o oceano quanto sobre o continente; no último caso, a discriminação do sinal de espalhamento é realizada usando apenas a diferença entre um canal de baixa freqüência (AMSU-A 23,8 GHz) e canais em alta freqüência (89 ou $150 \mathrm{GHz}$ ). Para situações livres de espalhamento, a diferença é aproximadamente zero, porém para situações com espalhamento, dependendo da quantidade total de partículas de gelo com tamanho de espalhamento no campo de visada do sensor, a diferença pode passar de 50 K. Grody et al. (1999), apresentaram um algoritmo para identificar a ocorrência de espalhamento em pixels do instrumento AMSU-A. Nesta técnica, desenvolvida originalmente para o SSM/I e SSM/T2, os índices de espalhamento são calculados a partir do valor da diferença entre medidas em alta freqüência $(89 \mathrm{GHz})$ e em canais de baixa freqüência (23 ou $31 \mathrm{GHz}$ ). Na ausência de nuvens, a temperatura de brilho geralmente aumenta com a freqüência e a diferença será sempre negativa; entretanto, na presença de espalhamento, a temperatura de brilho em $89 \mathrm{GHz}$ diminui, fazendo com que esta diferença se torne positiva. O índice de espalhamento foi calculado a partir das seguintes expressões:

$$
\begin{gathered}
\mathrm{SIL}=\mathrm{Tb}(23)-\mathrm{Tb}(89), \\
\mathrm{SIW}=113,2+[2,41-0,0049 * \mathrm{~Tb}(23)] * \mathrm{~Tb}(23)+ \\
0,454 * \mathrm{~Tb}(31)-\mathrm{Tb}(89),
\end{gathered}
$$

em que:

SIL e SIW são os índices de espalhamento sobre continente e oceano, respectivamente, e $\mathrm{Tb}(23), \mathrm{Tb}(31)$ e $\mathrm{Tb}(89)$ são as temperaturas de brilho observadas nas freqüências de 23, 31 e $89 \mathrm{GHz}$, respectivamente. Para valores de SIL $\geq 3$ e SIW $\geq 9$, o pixel é considerado contaminado por espalhamento.

O algoritmo apresentado acima é aplicável sobre o oceano tanto quanto sobre o continente, porém sobre o continente outros testes devem ser aplicados para detectar a presença de gelo e desertos, visto que esses tipos de superfície produzem uma quantidade de espalhamento que pode ser confundida com aquela produzida por hidrometeoros. Sobre o oceano, além do teste de espalhamento, aplica-se também outro, baseado na emissão em baixa freqüência para estimar a quantidade de água liquida nas nuvens (CLW). Uma formulação simples para derivação de CLW (Grody et al., 1999) é expressa por:

$$
\begin{gathered}
\mathrm{CLW}=(\mathrm{A}+\mathrm{B} * \log (285,0-\mathrm{Tb}(23))+ \\
\mathrm{C} * \log (285,0-\mathrm{Tb}(31))) * \cos \theta,
\end{gathered}
$$




$$
\begin{aligned}
& \mathrm{A}=8,240-(2,622-1,846 * \cos \theta) * \cos \theta \\
& \mathrm{B}=0,754, \\
& \mathrm{C}=-2,265
\end{aligned}
$$

em que:

$\theta$ é o ângulo zenital, e $\mathrm{Tb}(23)$ e $\mathrm{Tb}(31)$ as temperaturas de brilho observadas nas freqüências 23 e $31 \mathrm{GHz}$, respectivamente. Valores de CLW maiores que $0,3 \mathrm{~mm}$ geralmente estão associados à presença de precipitação, enquanto os abaixo deste limiar podem ser considerados livres de precipitação; no entanto, valores acima de $0,1 \mathrm{~mm}$ já são suficientes para contaminar os canais AMSU; portanto, como o interesse deste trabalho não é obter estimativa de precipitação mas identificar os pixels que possam estar contaminados por espalhamento, é mais apropriado utilizar-se o limite de $0,1 \mathrm{~mm}$, pois mesmo que o índice não seja suficiente para produzir precipitação, ele pode sê-lo para contaminar os canais.

A discriminação de regiões contaminadas por emissão e/ ou espalhamento na faixa de microondas, é bastante dependente da resolução espacial do sensor. Em uma área muito grande, o efeito pode ser mascarado e fica difícil ser identificado através de técnicas de detecção usuais; portanto, os sinais de espalhamento detectados na resolução do sensor AMSU-B $(15 \times 20 \mathrm{~km})$, podem não ser detectados na resolução do sensor AMSU-A (50x50 km). Diferentes combinações podem ser usadas entre os canais de superfície do AMSU-A e AMSU-B para se obter um índice de espalhamento mais preciso. Uma simples mudança sugerida por Bennartz et al. (1999), propõe trocar o canal em $89 \mathrm{GHz}$ do AMSU-A pelo canal em $89 \mathrm{GHz}$ do AMSU-B. Ferraro et al. (2000) propõem o cálculo de um índice de espalhamento sobre o continente, a partir da diferença entre os canais de 89 e $150 \mathrm{GHz}$ do AMSU-B, expressa por:

$$
\mathrm{SI}(150)=\mathrm{Tb}(89)-\mathrm{Tb}(150)
$$

De acordo com Ferraro et al. (2000), os pixels com índice $\mathrm{SI}(150) \geq 3 \mathrm{~K}$ são classificados como contaminados por espalhamento. Este teste foi originalmente concebido para trabalhar sobre o continente, porém também pode ser adaptado para o oceano.

Para se definir se um pixel sofre ou não a influência da presença de nuvens associadas a espalhamento e/ou emissão, realiza-se uma seqüência de testes baseados no índice de espalhamento e na CLW em cada pixel observado. De acordo com os resultados, cada pixel poderá ser classificado como limpo ou nublado (do ponto de vista da radiometria microondas). Os limiares aplicados neste algoritmo foram obtidos a partir da literatura (Bennartz et al., 1999; Ferraro et al., 2000; Grody et al., 1999) e por meio da comparação visual com imagens de satélite. Segundo a metodologia proposta, para que um pixel seja considerado limpo (livre de espalhamento e/ ou precipitação), as seguintes condições devem ser satisfeitas: a) se o índice de espalhamento para as freqüências de 23,8 , 31,4 e $89,0 \mathrm{GHz}$ for menor que $6,0 \mathrm{~K}$ sobre o oceano e menor que 3,0 K sobre o continente (Eqs. 1 e 2, respectivamente); b) se a mesma condição do item anterior for satisfeita, porém utilizando o canal de $89,0 \mathrm{GHz}$ do sensor AMSU-B (maior resolução espacial); c) se o índice de espalhamento para altas freqüências (89,0 e 150,0 GHz) for menor que $3 \mathrm{~K}$ (Eq. 4); este teste é realizado somente sobre continente, e d) se a CLW for menor que $0,1 \mathrm{~mm}$ (Eq. 3).

Uma das maiores dificuldades encontradas no processo de validação do algoritmo de detecção de nuvens é o estabelecimento de um critério objetivo e absoluto de comparação, uma vez que a maioria da informação relacionada à cobertura de nuvens provém de dados de satélite e está sujeita aos mesmos tipos de erro e imprecisão. No presente estudo, optou-se pela aplicação de uma análise subjetiva, como é comumente encontrado na literatura (França \& Cracknell, 1995). Basicamente, o processo de validação é feito através de uma comparação visual entre a máscara de espalhamento resultante da metodologia e imagens de satélite geradas pelo instrumento AVHRR em sua resolução plena (aproximadamente $1 \mathrm{~km}$ ) e também do sondador AMSU-B (16 km de resolução). Foram usadas as imagens dos canais 1 e 4 do AVHRR e dos canais 2 e 5 do AMSU-B. O canal 2 do AMSU-B $(150 \mathrm{GHz})$ está localizado na região de janela atmosférica, e o canal 5 (183 \pm 7 $\mathrm{GHz}$ ) possui o máximo da função peso (região de maior contribuição para a radiância medida) localizado próximo à superfície. A vantagem de se usar os canais AMSU-B está na sua alta (do ponto de vista de microondas) resolução espacial, que é aproximadamente 4 vezes maior que a do AMSU-A.

$\mathrm{O}$ algoritmo de máscara de espalhamento foi implementado no modelo de sondagem atmosférica Inversion Coupled with Image (ICI) (Lavanant et al., 1999). O impacto que a utilização da máscara de espalhamento apresentou sobre a recuperação dos perfis verticais de temperatura e umidade na atmosfera, foi avaliado para o período de 23 de fevereiro a 8 de março de 2000 (perfazendo o total de 32 imagens NOAA). As imagens foram geradas utilizando-se o software livre Generic Mapping Tools (GMT) (Wessel \& Smith, 1998).

\section{RESULTADOS E DISCUSSÃO}

É analisada a performance do algoritmo de identificação de pixels contaminados por espalhamento e/ou precipitação nos canais AMSU (microondas), que foi implementado no modelo de inversão ICI. Embora a análise tenha sido feita para várias imagens, preferiu-se apresentar os resultados de dois casos considerados representativos das demais situações; no primeiro caso, a maior parte da área coberta pela passagem do satélite sobre o Brasil encontra-se sobre o oceano Atlântico Sudeste e, no segundo, a maior parte se localiza sobre o continente possibilitando, assim, o uso de diferentes algoritmos. Ambos os casos apresentam várias situações em que ocorrem efeitos de espalhamento.

A Figura 1A mostra o índice de espalhamento (SI) calculado a partir dos canais 21, 22 e 36 (Eqs. 1 e 2) e a Figura 1B, o índice de espalhamento calculado a partir dos canais 36 e 37 (89 e 150 $\mathrm{GHz}$ ) na resolução espacial do instrumento AMSU-B (Eq. 4). O CLW obtido a partir da Eq. 3, é mostrada na Figura 1C, e o resultado da máscara de precipitação/espalhamento calculada com base nos limites descritos na Seção 2, está na Figura 1D. Os pixels (na grade do sensor HIRS) identificados como sendo contaminados por precipitação/espalhamento ou cujos valores 
A.

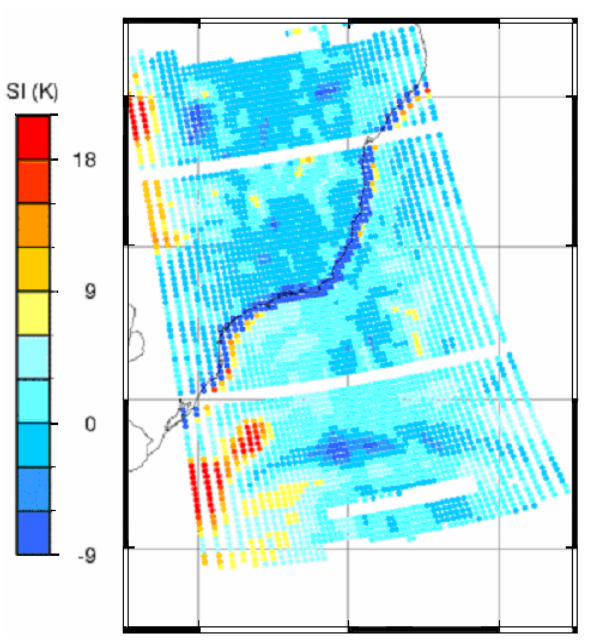

C.

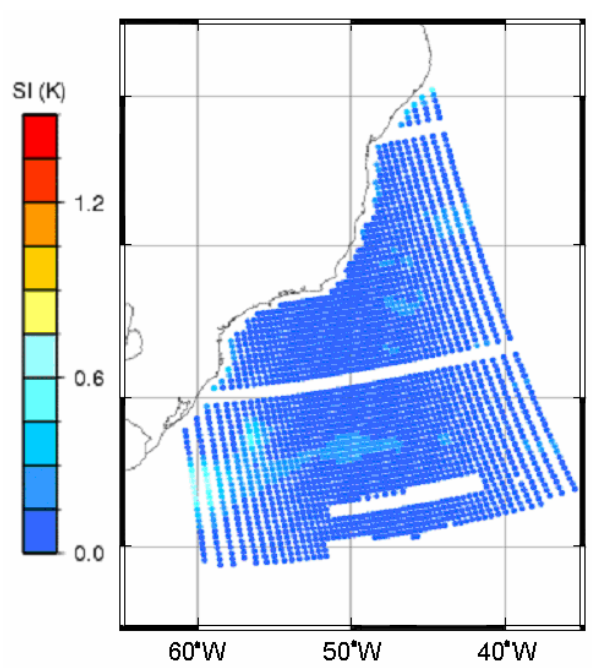

B.

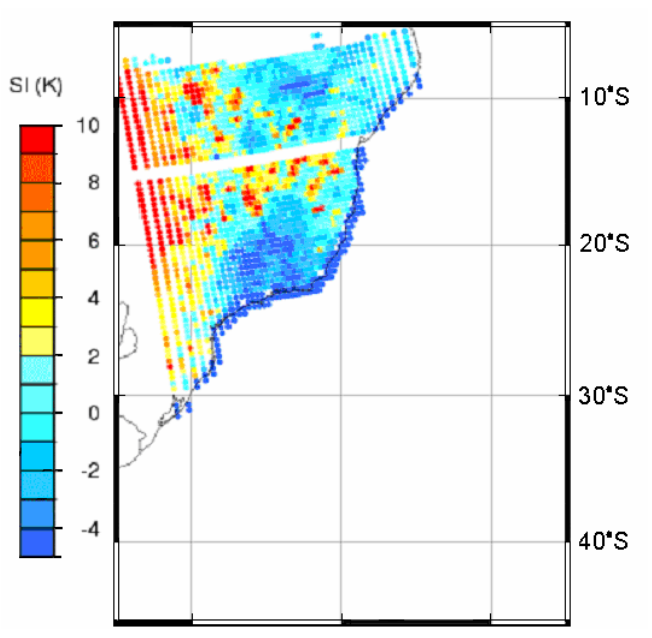

D.

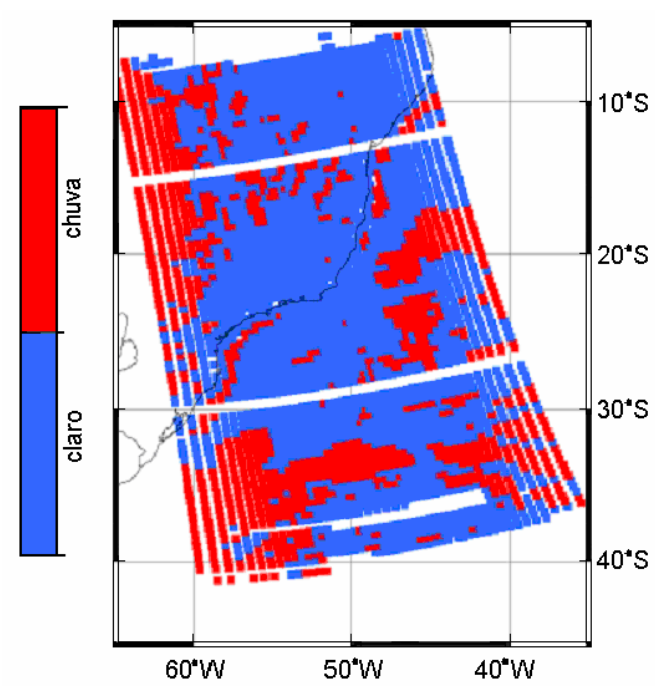

Figura 1. Processamento da imagem do satélite NOAA-15 do dia 02/03/2000, às 22:26 UTC: (A) Índice de espalhamento usandose os canais em 23,8, 31,4 e 89,0 GHz; (B) Índice de espalhamento empregando-se os canais em 89,0 e 150,0 GHz, (C) Quantidade total de água líquida nas nuvens, e (D) Máscara de nuvens (os pixels contaminados por espalhamento e/ou precipitação estão representados em vermelho)

A.

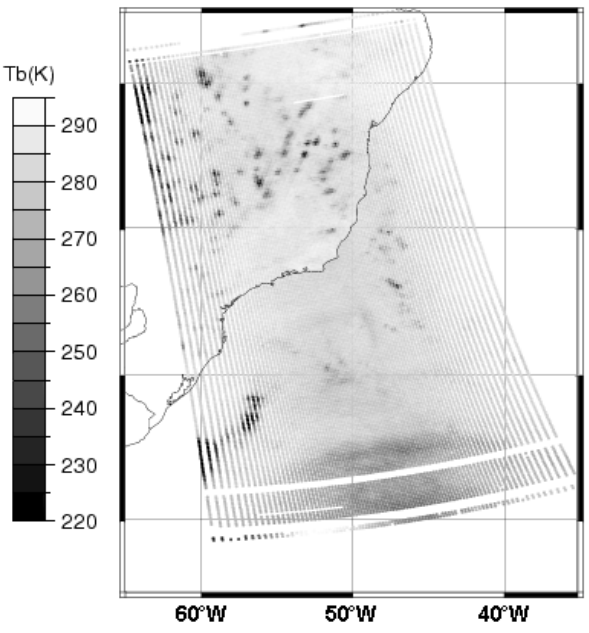

B.

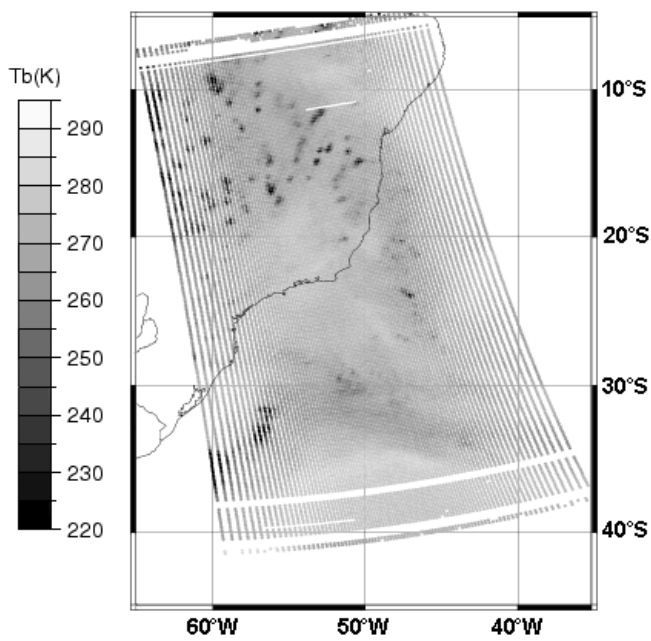

Figura 2. Imagens obtidas pelo NOAA-15, dia 02/03/2000, às 22:26 UTC (órbita 9375): (A) Temperatura de brilho do canal 2 (150 $\mathrm{GHz})$ do AMSU-B, e (B) Temperatura de brilho do canal $5(183 \pm 7 \mathrm{GHz})$ do AMSU-B 
de CLW ultrapassam o limite estabelecido, estão representados em vermelho na imagem. Comparando-se a máscara de nuvens (Figura 1D) com a imagem do canal 2 do AMSU-B (Figura 2A) nota-se forte correspondência entre as temperaturas de brilho relativamente mais baixas, geralmente associadas ao efeito de espalhamento por partículas de chuva e gelo nas nuvens, e as áreas identificadas como contaminadas por espalhamento, segundo metodologia utilizada (em vermelho). A mesma relação pode ser verificada quando a máscara de nuvens é comparada com o canal 5 do AMSU-B (Figura 2B), menos sensível aos parâmetros de superfície. Se a comparação for feita com a imagem no espectro do infravermelho (Figura 3) notar-se-á, também, uma grande correspondência entre áreas mais claras (temperatura de brilho muito baixa), indicando a presença de nuvens com topo elevado, em geral associado a regiões com precipitação (não se aplica para nuvens tipo cirros) e as áreas classificadas como sendo sujeitas a espalhamento, segundo o algoritmo.

As Figuras 4A-D mostram o resultado do segundo caso analisado (passagem diurna), no qual foi aplicado o algoritmo

A.

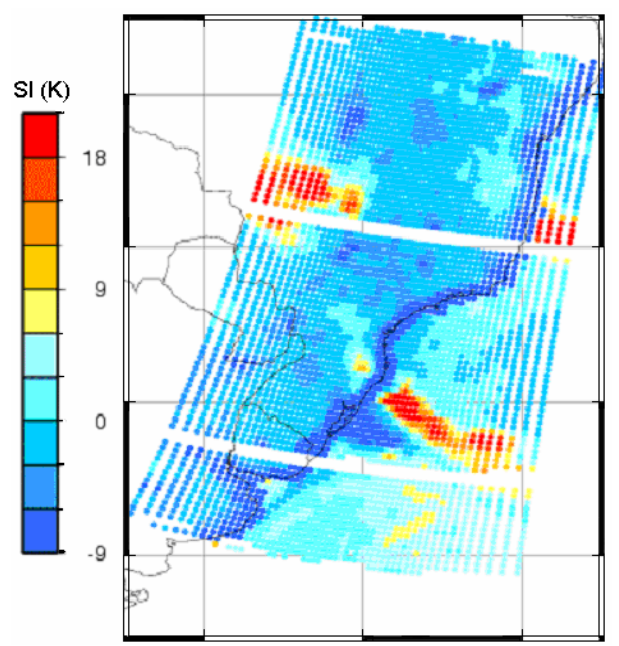

C.

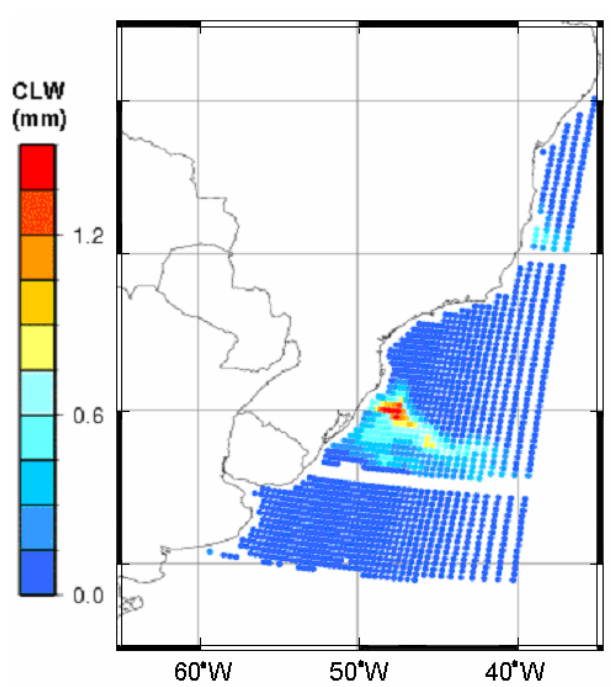

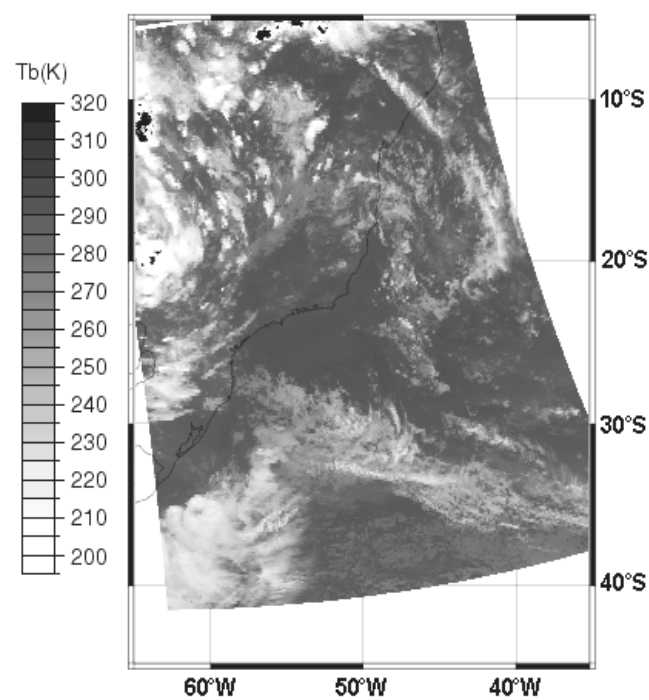

Figura 3. Temperatura de brilho observada no canal 5 do AVHRR $(12 \mu \mathrm{m})$. Imagem obtida pelo NOAA-15, dia 2/3/2000, às 22:26 UTC (órbita 9375)

B.

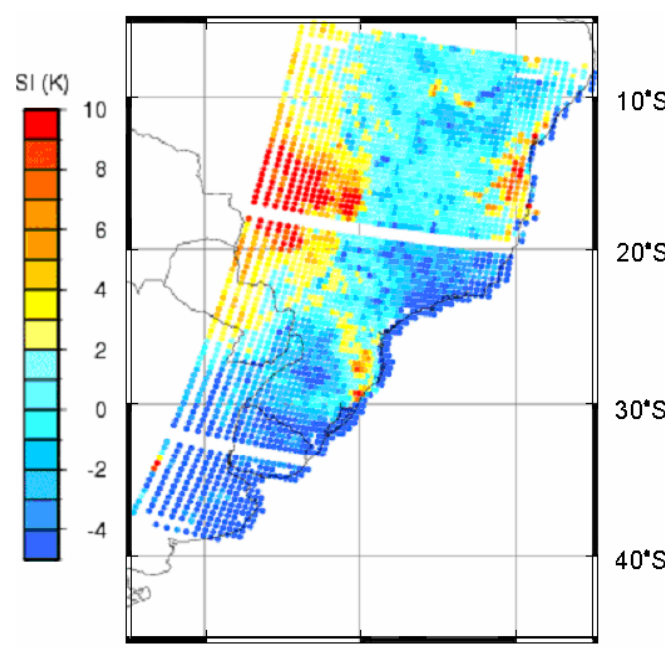

D.

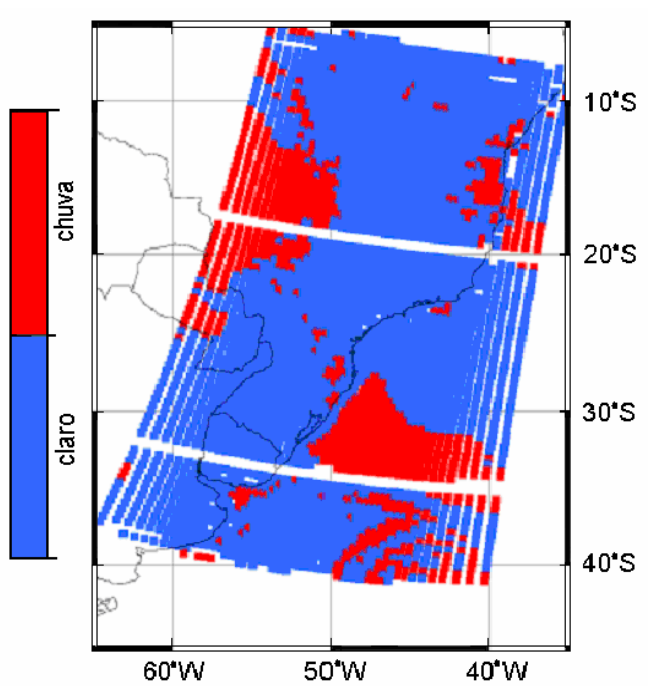

Figura 4. Processamento da imagem do satélite NOAA-15 do dia 01/03/2000, às 10:23 UTC: (A) Índice de espalhamento, empregandose os canais em 23,8,31,4 e 89,0 GHz; (B) Índice de espalhamento usando os canais em 89,0 e 150,0 GHz; (C) Quantidade total de água líquida nas nuvens, e (D) Máscara de nuvens (os pixels contaminados por espalhamento e/ou precipitação, estão representados em vermelho) 
de máscara de precipitação/espalhamento. As Figuras 5A e B ilustram as imagens dos canais 2 e 5 do AMSU-B, respectivamente, e a Figura 6A indica a imagem no espectro do infravermelho (canal 5 do AVHRR). Foi utilizada também, na comparação, a imagem do espectro visível (Figura 6B). Nesta órbita, o efeito de espalhamento ocorreu de forma concentrada em algumas regiões, como é o caso da região oceânica adjacente ao litoral Sul do Brasil, onde havia a presença de um sistema frontal; nesta imagem, todas as regiões correspondentes às áreas com nuvens com topos relativamente mais frios, foram identificadas pelo algoritmo. Um resultado interessante pode ser visto na área mais escura (temperatura de brilho mais baixa) na parte inferior da Figura 5A, que não está associada a efeitos de espalhamento, pois não se verifica a presença de nuvens nesta região (Figura 6A); neste caso, o algoritmo identificou corretamente a região como livre de espalhamento.
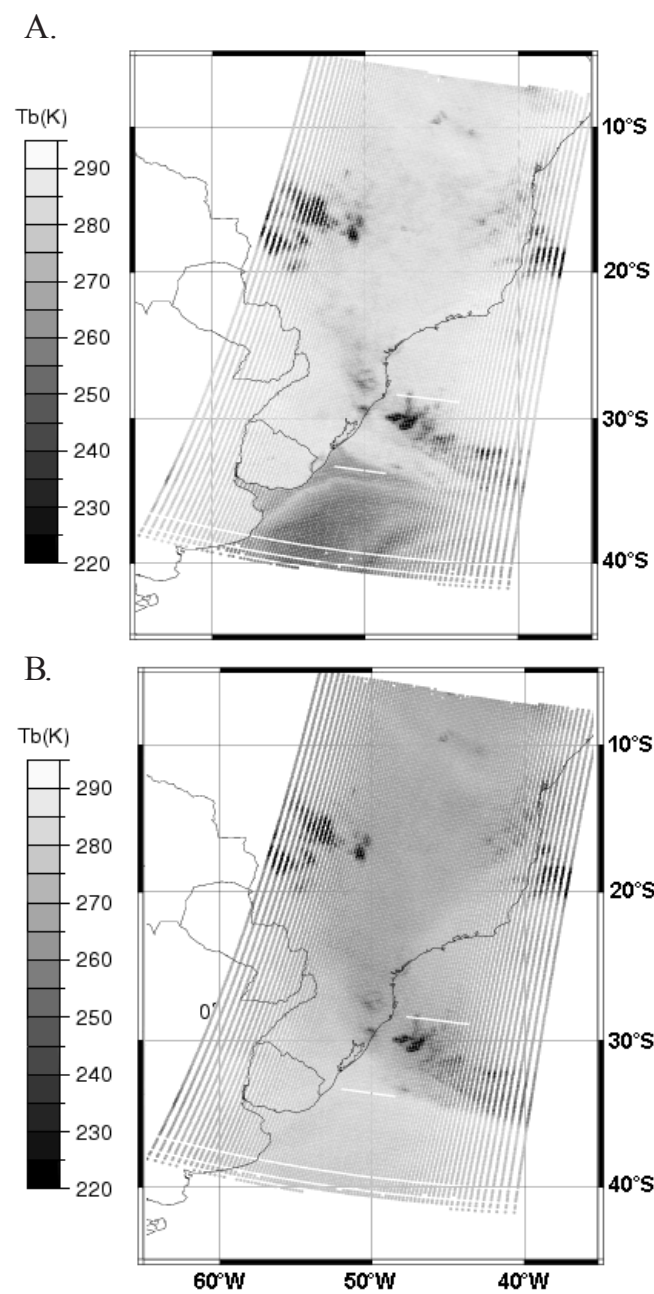

Figura 5. Imagens do satélite NOAA-15, para o dia 1/3/2000, às 10:23 UTC (órbita 9354): (A) Temperatura de brilho do canal

$2(150 \mathrm{GHz})$ do AMSU-B e (B) Temperatura de brilho do canal $5(183 \pm 7 \mathrm{GHz})$ do AMSU-B

Perfis verticais de temperatura e umidade sobre parte do Brasil e costa oeste do Atlântico Sul (região de cobertura das órbitas NOAA de Cachoeira Paulista) foram inferidos com o modelo de sondagem ICI. Os perfis foram agrupados segundo o resultado da classificação da máscara de espalhamento: perfis
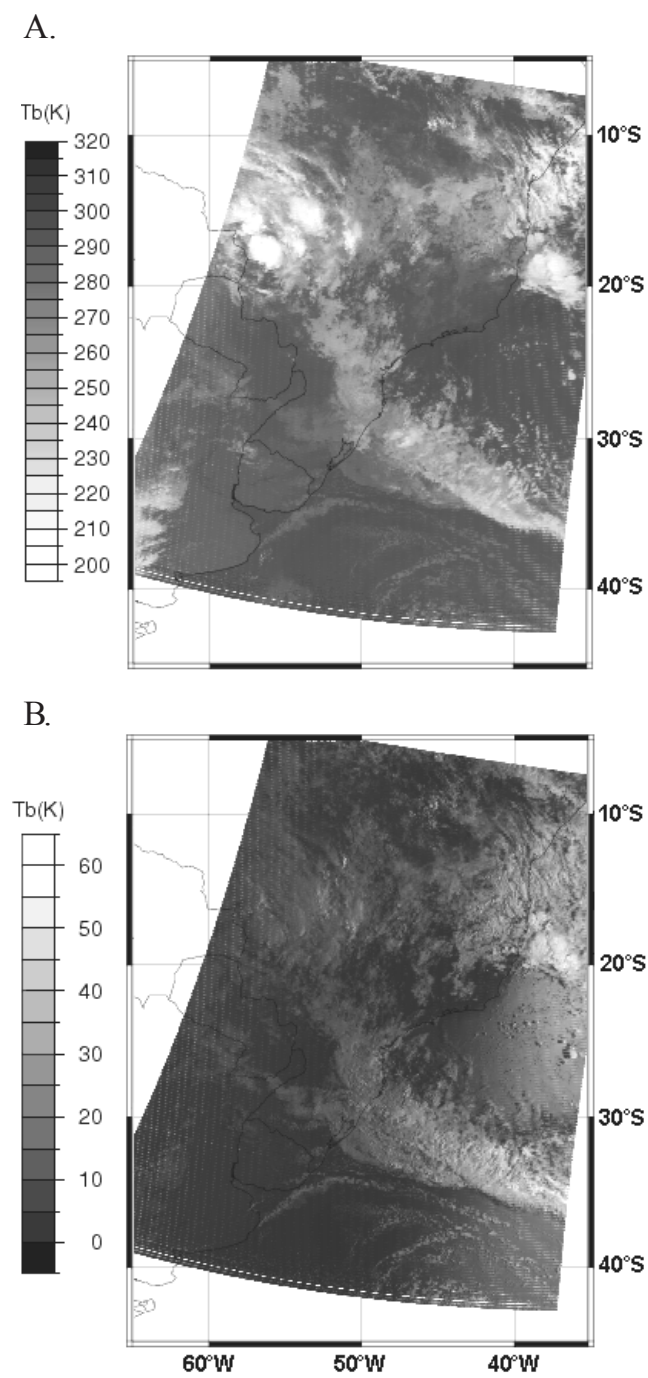

Figura 6. Imagens do satélite NOAA-15, para o dia 1/3/2000, às 10:23 UTC (órbita 9354): (A) Temperatura de brilho do canal 5 do AVHRR $(12 \mu \mathrm{m})$ e (B) Refletância do canal 1 do AVHRR $(0,6 \mu \mathrm{m})$

provenientes de pixels considerados contaminados por espalhamento nos canais microondas (TIPO 2), e perfis provenientes de pixels considerados limpos (TIPO 1). A Tabela 1 mostra uma estatística do erro entre os perfis inferidos pelo satélite e perfis provenientes de análise do modelo global do Centro de Previsão e Estudos Climáticos. Nota-se que o desvio padrão da diferença e o erro RMS são significativamente maiores para os perfis do TIPO 2. Os perfis do TIPO 2 apresentaram erro RMS 5.6\% maior que os do TIPO 1 para temperatura, e $4,4 \%$ para a umidade. Este aumento na estatística do erro para os perfis do TIPO 2 sugere a necessidade de se aplicar uma máscara de espalhamento no dados (como a que foi proposta neste trabalho) em aplicações tipo assimilação de dados, em modelos de previsão de tempo.

Uma avaliação geral dos dois casos apresentados e, também, de outros, não mostrados aqui, permitiu que se concluise que o algoritmo para identificação de pixels sujeitos a precipitação e/ou espalhamento, mostrou bom desempenho sobre a região em estudo, identificando praticamente todas as regiões contaminadas. Em geral, o algoritmo mostrou-se 
Tabela 1. Comparação entre perfis inferidos por satélite (modelado) e fornecidos pela análise do CPTEC (observado). Os cálculos foram efetuados para pixels considerados limpos pela máscara de espalhamento (TIPO 1), e perfis considerados contaminados pela máscara de espalhamento (TIPO 2)

\begin{tabular}{|c|c|c|c|c|}
\hline \multirow[t]{2}{*}{ Estatística } & \multicolumn{2}{|c|}{$\begin{array}{l}\text { Temperatura }(\mathrm{K}) \\
(1000 \text { a } 10 \mathrm{hPa})\end{array}$} & \multicolumn{2}{|c|}{$\begin{array}{l}\text { Umidade }\left(\mathrm{g} \cdot \mathrm{kg}^{-1}\right) \\
(1000 \text { a } 10 \mathrm{hPa})\end{array}$} \\
\hline & Tipo 1 & Tipo 2 & Tipo 1 & Tipo 2 \\
\hline N. de Perfis & 1381 & 643 & 1381 & 643 \\
\hline Desvio Padrão & 1,123 & 1,193 & 0,520 & 0,525 \\
\hline Erro RMS & 0,859 & 0,908 & 0,388 & 0,405 \\
\hline
\end{tabular}

bastante conservador, classificando pixels limpos (sem a presença de precipitação/espalhamento) como contaminados, sobretudo sobre o oceano, mas para o propósito a que essas informações são destinadas (inferência de perfis verticais de temperatura e umidade), é melhor que alguns pixels limpos sejam classificados erroneamente como contaminados que o contrário. Foi constatado neste trabalho, também, que em experimentos nos quais se utilizaram dados simulados (English, 1998; Burns et al., 1997), os pixels contaminados causam aumento considerável do erro no processo de inversão de perfis de temperatura e umidade.

\section{CONCLUSÕES}

1. O algoritmo proposto para identificação de pixels sujeitos a precipitação e/ou espalhamento nos canais AMSU mostrou bom desempenho sobre a região em estudo, identificando todas as regiões contaminadas.

2. Em geral, o algoritmo se mostrou conservador, classificando parte de pixels limpos (sem a presença de espalhamento) como contaminados, sobretudo sobre o oceano.

3. Os perfis verticais inferidos a partir de pixels considerados contaminados pelo algoritmo, apresentaram erro maior que aqueles inferidos a partir de pixels limpos. Para o erro RMS, a diferença foi de $5,6 \%$ para temperatura e de $4,4 \%$ para a umidade.

\section{AGRADECIMENTOS}

Os autores agradecem aos revisores anônimos que contribuíram significativamente para o aprimoramento deste trabalho, ao CPTEC/INPE, CNPq e CMS/Meteo-France, pelo apoio, e a Letícia Maria Barbosa de Faria, pela preparação das figuras.

\section{LITERATURA CITADA}

Bennartz, R.A.; Thoss, A.; Dybbroe, D.; Michelson, D.B. Nowcastng SAF: Precipitation analysis from AMSU. Visiting Scientist Report of Swedish Meteorological and Hydrological Institute (SMHI), Norrköping, Sweden, 1999. 36p.

Burns, A.; Wu, X.; Diak, G.R. Effects of precipitation and cloud ice on brightness temperatures in AMSU moisture channels. IEEE Transactions on Geosciences and Remote Sensing, New Jersey, v.35, n.6, p.1429-1437, 1997.
Carvalho, J.C. Modelagem e análise de sondagens remotas sobre o Brasil utilizando-se o sistema ICI. São José dos Campos: INPE, 2002. 230p. Tese Doutorado

Carvalho, J.C.; Ramos, F.M.; Ferreira, N.J.; Campos Velho, H.F. Retrieval of vertical temperature profiles in the atmosphere. In: 3rd International Conference on Inverse Problems in Engineering, Port Ludlow, Proceedings of 3ICIPE, USA, 1999. CD-Rom

Chalfant, M.; Reale, A. Status of NOAA Advanced Microwave Sounding Unit-B (AMSU-B) products. In: Technical Proceedings of the Tenth International TOVS Study Conference, Boulder: 1999. 1999,

English, S.J. Estimation of temperature and humidity profile information from microwave radiances over different surface types. Journal of Applied Meteorology, Boston, v.38, n.20, p.1526-1541, 1999.

English, S.J.; Hewison, T.J. A fast generic millimetre-wave emissivity model. Microwave Remote Sensing of the Atmosphere Environment, Proceedings of SPIE, London, v.3503, p.288-299, 1998.

English, S. J.; Jones, D.C.; Dibben, P.C.; Renshaw, R.J.; Eyre, J.R. The impact of cloud and precipitation on ATOVS soundings. ECMWF report, London, 1999b.

Ferraro, R.; Weng, F.; Grody, N.C.; Zhao, L. Precipitation characteristics over land from the NOAA-15 AMSU sensor. Geophysical Research Letters, v.27, n.17, p.2669-2672, 2000.

França, G.B.; Cracknell, A.P. AVHRR daytime data masking approach using NOAA AVHRR daytime data for tropical areas. International Journal of Remote Sensing, London, v.16, n.9, p.1697-1705, 1995.

Goodrun, G.; Kidwell, K.B.; Winstons, W. NOAA KLM user's guide. Washington, D.C.: NOAA/NESDIS/NCDC/CSD/SSB, 2001.

Grody, N; Weng, F.; Ferraro, R. Application of AMSU for obtaining water vapour, cloud liquid water, precipitation, snow cover and sea ice concentration. Technical Proceedings of the Tenth International TOVS Study Conference, Boulder, 1999.

Klaes, K.D. ATOVS data processing in Europe. Technical Proceedings of the Ninth International TOVS Study Conference, Igls, Austria, p.267-274, 1997.

Klaes, K.D; Schraidt, R. The European ATOVS and AVHRR Processing Package (AAPP) Development. Technical Proceedings of the Tenth International TOVS Study Conference, Boulder, 1999, p.288-294

Labrot, T.; Lavanant, L.; Whyte, K. General specifications for the AAPP pre-processing package related to NOAA polar orbiting weather satellites; AAPP Documentation - Scientific Part, Meteo France. http://.www.met-office.gov.uk/research/ interproj/nwpsaf/atovs/aapp3_s_025.pdf, 25 ago. 2003.

Lavanant, L.; Brunel, P.; Rochard, G.; Labrot, T. NOAA15 Soundings profiles retrieved with the ICI scheme. Technical Proceedings of the Tenth International TOVS Study Conference, Boulder, 1999. p.312-328

Smith, W.L. Atmospheric Soundings from satellite - false expectation or the key to improved weather prediction? Quarterly Journal of Royal Meteorological Society, London, v.117, n.498, p.267-297, 1991.

Wessel, P.; Smith, W.H.F. New, improved version of Generic Mapping Tools released, EOS Transactions, AGU, Washington D.C., v.79, n.47, p.579, 1998. 